\title{
MICROWAVE-ASSISTED REGENERATION OF ACTIVATED CARBONS LOADED WITH PHARMACEUTICALS
}

C.O. Ania*, J.B. Parra, J.A. Menéndez, J. J. Pis

Instituto Nacional del Carbon, P.O. Box 73, 33080 Oviedo, Spain

*To whom correspondence should be addressed;

C.O. Ania, e-mail: conchi@incar.csic.es

\begin{abstract}
The purpose of this work was to explore the application of microwaves for the regeneration of activated carbons spent with salicylic acid, a metabolite of a common analgesic frequently found in wastewater from pharmaceutical industry. The exhausted carbon was treated in a quartz reactor by microwave irradiation at $2450 \mathrm{MHz}$ at different temperature and atmosphere, being the regeneration efficiency highly dependent on the operating conditions. Quantitative desorption of the pollutant was achieved at high temperature and oxidizing atmosphere, with regeneration efficiencies as high as $99 \%$ after 6 cycles. The stripping efficiency was superior to $95 \%$ at high temperatures and decreased at $450{ }^{\circ} \mathrm{C}$. The incomplete desorption of the adsorbate at low temperature was further confirmed by the changes in the porosity observed by $\mathrm{N}_{2}$ and $\mathrm{CO}_{2}$ adsorption isotherms. Hence, micropores remain blocked which turns out in a reduction in loading capacities in successive cycles.
\end{abstract}

Keywords: microwave regeneration, activated carbon; pharmaceuticals; wastewater 


\section{Introduction}

During the last three decades, research on the impact of chemical pollution has focused almost exclusively on the so-called "conventional" persistent organic pollutants (i.e., POPs) and a number of reviews studies can be found in the literature (Bandosz, 2006; Radovic et al., 2000). Recently, considerable interest has arisen regarding the occurrence of emerging pollutants such as pharmaceuticals, hormones, household chemicals and other extensively used POPs in water resources (Ferrari et al., 2004). This concern is understandable given the risk they pose to the environment and to human health (Jones et al., 2005; Ferrari et al., 2004). Research has shown that these xenobiotics can enter in water streams through different pathways, disperse, and persist to a greater extent than first anticipated.

Since 1980s a number of new POPs have been identified in surface and ground water, and drinking water (Kolpin et al., 2002). This is extremely important if one bears in mind that drinking water provides a direct route into the body for any drugs that might be present. Information on the environmental hazard of the majority of pharmaceuticals is scarce; hence the possible adverse impact of these residues remains still uncertain and under debate (Hensely and Murray, 2000; Aygscough et al., 2000).

Moreover, it has been reported that many pharmaceuticals may be unaffected or only partially removed from wastewater during biological processes in sewage treatment facilities with their consequent release into surface waters. In other cases, the municipal treatment plants simply are not designed to remove these refractory compounds from the effluent (Halling-Sorensen et al., 1998; Daughton and Ternes, 1999; Hernando et al., 2006). Therefore the adoption of advanced processes in existing sewage treatment facilities is critical (Ernst and Jekel, 1999; Vogna et al., 2004). In these lines, adsorption on activated carbons (AC) is a well established technology widely used in the waste 
water purification (Bandosz, 2006), which is supported by a high depuration efficiency and possibility of regeneration.

Common regeneration techniques of $\mathrm{AC}$ beds in industrial applications are based on thermal (steam, carbon dioxide or inert atmosphere) and chemical methods ( $\mathrm{pH}$-swing or extraction with solvents). However, regeneration is time and energy-consuming and very often there is a significant deterioration of the adsorbent's pore structure, thereby reducing the final adsorption capacity and the efficiency of the regeneration (Ania et al., $2004 a ; 2005)$. This has encouraged industries to seek for alternative methods to regenerate the exhausted adsorbents. An overview about regeneration methods can be found in (Bathen, 2003).

In recent years, microwave (MW) irradiation has attracted the attention as a tool for regeneration due to its capability of molecular level heating which leads to homogeneous and quick thermal reactions. Interesting reports have appeared on the application of microwave-heating technology for regenerating AC (Price and Schmidt, 1997; Bradshaw et al., 1997; Coss and Cha, 2000; Liu et al., 2004, 2006; Jones et al, 2002; Quan et al., 2004; Zhang-Steenwinkela et al., 2005), with very promising results. Microwave regeneration offers potential advantages over conventional treatment (Ania et al., 2004a, 2005), including energy savings, and better performance of the AC in terms of ulterior adsorption capacity and rate of adsorption (Coss and Cha, 2000; Cha and Carliste, 2001; Jones et al., 2002; Ku et al., 2002; Quan et al., 2004; Liu et al., 2004, 2006; Zhang-Steenwinkela et al., 2005). Consequently, microwave technology is gaining importance in several fields of application on an industrial scale, being considered a promising available technology for the regeneration of carbon materials.

The purpose of this study was to investigate the regeneration of activated carbons used in the purification of pharmaceutical wastewaters by means of MW irradiation. To this 
end, successive adsorption-regeneration cycles were carried out in a single mode microwave furnace at the laboratory scale. The efficiency of regeneration was evaluated, and the changes in the properties of the adsorbent after each regeneration step were investigated, and linked with the adsorption capacities. The object of the study was a high-volume pharmaceutical compound (salicylic acid), a primary hydrolytic metabolite of acetylsalicylic acid, which is present in wastewater resulting from the industrial production of a common analgesic.

\section{Experimental Section}

\section{Materials}

A commercial powdered activated carbon -CV-, supplied by Bayer SA and obtained from chemical activation of wood with phosphoric acid was chosen for this study. This adsorbent is commonly used for the purification of industrial wastewater and drinking water. Before the experiments the as-received carbon was soaked in a Soxhlet apparatus with distilled water to remove soluble components, dried at $120^{\circ} \mathrm{C}$ overnight and stored in a desiccator until use.

\section{Saturation of the activated carbon beds}

Details on the experimental equipment used have been described elsewhere (Ania et al, 2004a). Briefly, rapid small-scale column tests were used for the evaluation of the adsorptive capacity. Breakthrough curves were obtained at $30^{\circ} \mathrm{C}$ in columns packed with ca. $5 \mathrm{~g}$ of activated carbon. All the experiments were conducted at an initial concentration of $2 \mathrm{~g} \mathrm{~L}^{-1}$, and a flow rate of $4 \mathrm{~cm}^{3} \mathrm{~min}^{-1}$. The outlet concentration was continuously monitored by means of a UV-VIS spectrophotometer at the corresponding wavelength. The adsorptive capacities were obtained by integrating over the entire breakthrough curve. When the saturation point was reached, the exhausted AC was 
washed with distilled water for $2 \mathrm{~h}$ to eliminate any excess of pollutant, and dried overnight at $120^{\circ} \mathrm{C}$ to reduce the moisture content. Afterwards, the sub-samples of the exhausted AC were regenerated.

\section{Regeneration of the exhausted activated carbon beds}

The procedure followed for the regeneration of $\mathrm{AC}$ has been described elsewhere (Ania et al, 2005a). Briefly, the MW device consists of a microwave magnetron of a maximum output power $2000 \mathrm{~W}$ at $2450 \mathrm{MHz}$ and a single mode cavity where the sample is exposed to microwave irradiation. An optical pyrometer was used to control the temperature. The exhausted samples were regenerated at $850^{\circ} \mathrm{C}$ under an inert $\left(\mathrm{N}_{2}\right)$ and oxidizing $\left(\mathrm{CO}_{2}\right)$ atmosphere, using a gas flow rate of $10 \mathrm{~cm}^{3} \mathrm{~min}^{-1}$. The inert atmosphere was maintained during the heating up and cooling-down intervals. Samples will be denoted as $\mathrm{CV}$ followed by a reference to the atmosphere of the regeneration $(\mathrm{N}$ for $\mathrm{N}_{2}$ and $\mathrm{C}$ for $\mathrm{CO}_{2}$ ), the temperature ( 4 for 450 and 8 for $850{ }^{\circ} \mathrm{C}$ ) and the number of regeneration cycles. For instance, sample CV N4 corresponds to CV activated carbon regenerated at $450{ }^{\circ} \mathrm{C}$ under inert atmosphere. The effect of the heating mechanism in the absence of the adsorbate was also evaluated; the blanks will be denoted as CVMW followed by the reference for the atmosphere and the temperature of the treatment (i.e., CVMW 4N).

The yield of regeneration was evaluated by means of the Regeneration efficiency (RE) and the single Step Stripping Efficiency (SSE) for the samples subjected to multiple regeneration steps. The $\mathrm{RE}$ was calculated as $\mathrm{RE}=\mathrm{Q}_{\mathrm{i}} / \mathrm{Q}_{\mathrm{o}} * 100$, where $\mathrm{Q}_{\mathrm{i}}$ the adsorptive capacity of the regenerated carbon and $\mathrm{Q}_{\mathrm{o}}$ the adsorptive capacity of the fresh carbon. In contrast, the SSE was defined as the ratio $\mathrm{SSE}=\mathrm{Q}_{\mathrm{i}} / \mathrm{Q}_{\mathrm{i}-1} * 100, \mathrm{Q}_{\mathrm{i}-1}$ being the adsorptive capacity of a previous regeneration cycle. The SSE parameter allows to obtain the yield 
of desorption of pollutant in the different cycles, enabling to study the evolution of the overall yield of regeneration.

\section{Textural and chemical characterization}

Textural characterization was carried out by measuring the $\mathrm{N}_{2}$ adsorption isotherms at $-196^{\circ} \mathrm{C}$ in an automatic apparatus (Micromeritics ASAP 2010M). Before the experiments, the samples were outgassed under vacuum at $120{ }^{\circ} \mathrm{C}$ overnight. The isotherms were used to calculate the specific surface area, $\mathrm{S}_{\mathrm{BET}}$, total pore volume, $\mathrm{V}_{\mathrm{T}}$, and pore size distributions. The pore size distributions were evaluated using the density functional theory (DFT). Additionally, the narrow microporosity (pore width smaller than $0.7 \mathrm{~nm}$ ) was estimated from $\mathrm{CO}_{2}$ adsorption isotherms at $0^{\circ} \mathrm{C}$ performed in a Micromeritics Gemini 2375, using $1.023 \mathrm{~g} \mathrm{~cm}^{-3}$ as the density of adsorbed $\mathrm{CO}_{2}$ and 0.36 as $\beta$ parameter. The as-received and regenerated samples were further characterized by ultimate analysis and the point of zero charge, $\mathrm{pH}$ PZC (Noh and Schwarz, 1992). Thermal analysis was also performed in the samples before and after salicylic acid adsorption (sat series), using a TA Instrument thermal analyzer. The instrument settings

were heating rate $10^{\circ} \mathrm{C} \mathrm{m^{-1 }}$ and nitrogen atmosphere with $100 \mathrm{~cm}^{3} \mathrm{~min}^{-1}$ flow rate. For each measurement about $25 \mathrm{mg}$ of carbon sample was used.

\section{Results and discussion}

The goal of this work was to investigate the effect of MW irradiation for the regeneration of activated carbons used in the purification of wastewater from pharmaceutical industry. The targeted pollutant (salicylic acid) is a valuable chemical in the industrial process of the preparation of a common analgesic. Therefore, its recovery and/or degradation upon MW heating will be studied, along with the effect on the 
loading capacity after subsequent cycles of regeneration and the textural properties of the activated carbon.

\subsection{Effect of MW regeneration on the uptake of salicylic acid}

As reported previously, the temperature reached when a given activated carbon is exposed to microwave irradiation depends on many factors and it can be modified by adjusting the input power on the MW device (Ania et al., 2005a). In this work, the temperature was fixed at 450 and $850{ }^{\circ} \mathrm{C}$; therefore, in order to maintain this value, the input power was gradually adjusted with the regeneration cycles. The exposure time of the activated carbon at the temperature of the regeneration (i.e., once the temperature has been reached) was 4 min. This value was set as the time necessary to attain a $3 \%$ degree of burn-off under a $\mathrm{CO}_{2}$ atmosphere, in order to compare the results with those obtained under oxidizing conditions, as discussed in previous works (Ania et al., 2004a).

The influence of thermal treatment in the MW device on the overall uptake of the raw carbon was initially investigated. Table 1 shows the adsorptive capacities for salicylic acid on CV carbon after successive regeneration cycles. For the sake of comparison, the capacities of the samples exposed to microwave heating in the absence of the adsorbate (non-saturated) are also included. It can be observed that when the carbon is exposed to microwaves, the adsorption capacity increased, which may be linked to changes induced in the microporous structure of the carbon, as it will be discussed below. This behavior is more remarkable at $850{ }^{\circ} \mathrm{C}$. Regarding the effect of the atmosphere, the uptake slightly increases under $\mathrm{CO}_{2}$, which is expected given that the carbon undergoes a slight activation at these operating conditions and taking into account that micropores are preferential sites of salicylic acid adsorption (Ania et al., 2004a). 
The trends observed in RE and SSE values with the cycles of regeneration are illustrated in Figures 1 and 2, respectively. The regeneration efficiency -RE- gradually decreases after several cycles, the extent of which strongly depends on the temperature and atmosphere. For instance, the regeneration is favored at high temperatures and under $\mathrm{CO}_{2}$ atmosphere (RE above $98 \%$ after 6 cycles), compared to inert atmosphere ( $88 \%$ in the sixth cycle), due to partial gasification of the adsorbent. The reduction in the yield of regeneration becomes particularly important at low temperature (i.e., $450{ }^{\circ} \mathrm{C}$ ) after six cycles, with a RE below $65 \%$. Moreover, under the same atmosphere (i.e., $\mathrm{N}_{2}$ ) desorption of salicylic acid is more quantitative at high temperatures. This is expected since the amount of energy supplied to the sample increases with temperature. It is worth noting that the loading capacity of salicylic acid exceeded its initial value when regeneration was carried out at $850{ }^{\circ} \mathrm{C}$ under oxidizing atmosphere; the same trend was maintained for several cycles. It should be kept in mind that a similar behavior had also been observed after MW irradiation of the non-saturated carbon, which suggests that this might be linked to the structural changes induced in the carbon. For this reason, the enhancement in the regeneration efficiency may not be exclusively attributed to the complete desorption of the adsorbate, but also to an increase in the overall uptake linked with the alterations of the adsorbent.

Conversely, the stripping efficiency SSE was very high, indicating that complete desorption can be achieved under certain conditions. For instance, the SSE coefficient was superior to $99 \%$ at $850{ }^{\circ} \mathrm{C}$ in oxidizing atmosphere, and it remained somewhat constant for each series (Figure 2), Comparatively, SSE is lower at $450{ }^{\circ} \mathrm{C}$, confirming that desorption on every step is less quantitative at lower temperatures.

The low performance at $450{ }^{\circ} \mathrm{C}$ should be attributed to the incomplete desorption of the adsorbate. It is worthwhile to note that the carbon was regenerated for $4 \mathrm{~min}$ at the 
respective temperature of regeneration. It seems evident that such a short period of time is not enough to promote the quantitative desorption (particularly at $450{ }^{\circ} \mathrm{C}$ ) of the large amount of pollutant retained the carbon (adsorption capacity is $372 \mathrm{mg} \mathrm{g}^{-1}$, which represents ca. $37 \%$ in weight). Similar findings can be found in the literature (Liu et al., 2004; Ania et al, 2004a). As aforementioned, the conditions of the regeneration were fixed to attain a slight activation of the carbon under oxidizing atmosphere, and therefore a complete regeneration of the carbon bed is not necessarily attained.

During the MW heating, desorption of the adsorbate retained on the exhausted activated carbon was observed between $200-400{ }^{\circ} \mathrm{C}$ (change in the color of the evolved gases). The same range of temperature was observed in subsequent cycles of regeneration. This observation is in good agreement with the temperature of desorption obtained in the derivative thermogravimetric -DTG- profiles of the spent carbons (sat series) obtained in a thermobalance (Figure 3).

From the DTG curves it can be seen that a peak of high intensity and centered at around $260^{\circ} \mathrm{C}$ appears after salicylic acid adsorption. This peak can only be assigned the evolution of salicylic acid physisorbed in the micropores, since it was absent before adsorption. This was further supported by the identification of the evolved gases by TGMS. Identification was performed by analysis of mass spectra and verified by comparison with the MS database. Salicylic acid was the major compound, showing a variation of the relative abundance with temperature; other compounds identified were phenol and $\mathrm{CO}_{2}$. This seems logical since, according to literature, salicylic acid sublimes when gradually heated at about $200^{\circ} \mathrm{C}$, but after sublimation it has a tendency to spontaneously decompose into phenol and $\mathrm{CO}_{2}\left(\mathrm{O}^{\prime}\right.$ Neil et al., 2001).

In order to avoid a misinterpretation of the results, it must be stressed that the peaks in the DTG curves at temperatures above $500{ }^{\circ} \mathrm{C}$, are linked to the decomposition of the 
surface functionalities. These peaks are not further observed after the first regeneration cycle at high temperature (series $\mathrm{N} 8$ and $\mathrm{C} 8$ ) since it is known that the oxygen functionalities are removed by annealing (Ruiz-Martínez et al., 2006).

The absence of a second desorption peak at high temperatures, observed for instance in the case of some aromatics (i.e., phenol) (Ania et al., 2005b), indicates a lack of specific interactions between the surface functionalities and the salicylic acid molecule, despite the high degree of functionalization of this compound.

For a given series, the temperature of the desorption peak remained unchanged, regardless the number of regeneration cycles. In contrast, the DTG peak slightly shifted to higher temperatures for the series treated at $850{ }^{\circ} \mathrm{C}$. To understand this behavior the porous texture of the carbon was characterized after several regeneration cycles, and it is discussed below.

On the basis of these results it can be inferred that that microwave energy contributes to an increase in the efficiency of the regeneration of saturated AC, and in the life span of the activated carbon. Salicylic acid is quantitatively desorbed when high temperatures are applied. Besides, it does not undergo decomposition inside the porous structure of the carbon when exposed to MW irradiation. It appears to be desorbed in a molecular state and later decomposed in the gas phase on heating. This fact contrasts with the behavior obtained for other aromatics such as phenol, which partially decomposes inside the carbon matrix creating coke deposits that block the porosity of the carbon (Ania et al., 2004a; 2005b). As a result, better yields of regeneration are obtained in the carbon loaded with salicylic acid over a number of cycles. This finding is very important, not only from the point of view of the regeneration of the carbon, but also for the recovery of the pollutant. Both salicylic acid and phenol are valuable intermediate chemicals used in the fabrication of a common analgesic, for which their recovery for 
further reuse would be extremely economically attractive. Research on the possibility of recovery and reuse of salicylic acid and/or phenol (via condensation from the outlet gases) retrieved from the regeneration of the adsorbent is currently ongoing.

\subsection{Effect of MW regeneration on the textural properties of the carbon}

Detailed characteristics of the pore structure of the series of as-received and regenerated carbons are presented in Table 2 and Figure 4. Initially, the influence of thermal treatment in the MW device on the porosity of the as-received carbon was investigated. As aforementioned, adsorption capacities increased after MW irradiation of the adsorbent, which suggests that some textural changes are produced upon heating which are beneficial for the retention of salicylic acid.

The porous features of the non-saturated carbon after MW irradiation differ slightly from those of the parent $\mathrm{CV}$ carbon. In all cases, the nitrogen adsorption isotherms presented a type I with somewhat type IV character according to BDDT classification (Brunaeur, 1938), pointing to microporous carbons with a considerable development of mesoporosity. Although the shape of the isotherms was rather preserved, specific surface areas and micropore volumes slightly decreased after heating in the microwave device (Table 2). This behavior, which has been reported in the literature (Ania et al., 2005; Rao et al., 1996) is particularly remarkable when the carbon was heated at $850{ }^{\circ} \mathrm{C}$ under inert atmosphere. These changes in the porous structure in the absence of the adsorbate are attributed to internal reorganizations of the carbon as a consequence of the large amount of gases evolved during pyrolysis. This is expected in the case of CV sample due to its large oxygen content $(9.7 \%)$ and volatile matter $(22 \%)$. Upon heating, a large amount of gases -coming form desorption of volatiles and the decomposition of oxygen-containing groups- are evolved (even at $450{ }^{\circ} \mathrm{C}$ ). As a result, the porosity of the carbon is modified, being this effect more remarkable at $850{ }^{\circ} \mathrm{C}$ 
where the amount of gases evolved is much larger. This is likely connected to a slight activation of the carbon as a consequence of $\mathrm{CO}_{2}$ evolved.

This has important implications in the adsorption capacity in successive cycles, as it can be observed in Table 1. Despite the fall in the porosity, as observed by nitrogen, the adsorption capacity increased after MW irradiation. The analysis of the narrow microporosity of the samples throws some light over these a priori contradictory results. The $\mathrm{CO}_{2}$ data shows an effect of porosity opening during the treatment at $850{ }^{\circ} \mathrm{C}$ (see Figure 4 and Table 2). The amount of $\mathrm{CO}_{2}$ adsorbed at low relative pressures is larger for the MW irradiated samples than for CV carbon, which is indicative of the presence of a large amount of narrow micropores. Furthermore, a shift towards micropores of smaller sizes occurred. This observation was further confirmed by the PSD obtained by application of the DR model to the $\mathrm{CO}_{2}$ adsorption isotherms. The volume of micropores increased after MW heating, indicating that the carbon is slightly activated. Taking into account the small dimensions of the adsorbate (ca. diameter $5.2 \AA$ and excluded molecular area $66.15 \AA^{2}$, evaluated from molecular simulation), it appears that increasing the volume of narrow micropores due to MW irradiation turns up in an enhancement of the adsorption capacity, underlining the important role of narrow microporosity in salicylic acid uptake. This finding is in good agreement with the mechanism of retention proposed for this compound in earlier works (Ania et al., 2004b). This fact also explains the upward shift in the temperature of desorption observed in the DTG profiles. The adsorption potential is higher in the microporosity of narrower sizes, therefore the temperature of desorption increase.

After salicylic acid adsorption, a slow and gradual fall in the porosity occurred due to some pore blocking upon regeneration, this being more evident after 3 and 6 cycles. The highest decrease in $\mathrm{CO}_{2}$ uptake after successive regeneration cycles corresponds to the 
sample treated at $450{ }^{\circ} \mathrm{C}$, which is in good agreement with the lower adsorptive capacities achieved in liquid phase. This effect is attributed to the damage in the porous structure (pore blockage) as a consequence of the incomplete desorption of the salicylic acid retained in the carbon.

In contrast, when regeneration is carried out at high temperature, adsorptive capacities after the second and third regeneration cycles were larger or similar to that in the asreceived carbon. With regards to microporosity, the reduction in the micropore volumes is smaller at high temperatures. It can be observed from the shape of the isotherms in Figure 4 that the amount of $\mathrm{CO}_{2}$ adsorbed at low relative pressures still exceeded that of the parent carbon, after several cycles of regeneration. This confirms that desorption of salicylic acid is more quantitative at $850^{\circ} \mathrm{C}$, although a small fraction of narrow micropores remained blocked.

In summary, the use of microwave-assisted techniques for the regeneration of activated carbons loaded with salicylic acid resulted in high regeneration yields over 6 regeneration cycles at high temperatures. Moreover, desorption of this compound is quantitative, enabling the possibility of recovery and reuse of this compound. Upon MW irradiation, an increase in the volume of narrow micropores occurred, which turned up into a lesser reduction in the salicylic acid uptake in subsequent cycles. Therefore, the adsorbent shows a superior performance and both RE and SSE coefficients are larger than the original activated carbon.

\section{CONCLUSIONS}

We have demonstrated that the use of microwave-assisted technology as a tool for the regeneration of activated carbons exhausted with pharmaceuticals has several advantages on the efficiency of the regeneration process: 
- The yield of regeneration highly depends on the operating conditions. Large regeneration efficiencies are achieved at high temperatures (above $95 \%$ after 6 cycles) and under oxidizing atmosphere due to partial gasification of the adsorbent. The single step stripping efficiency remains somewhat constant for each series, being superior at high temperatures.

- Adsorption capacities after MW irradiation of the as-received carbon exceeded the initial value of the raw material, due to changes in the microporous structure of the carbon upon heating.

- Salicylic acid is desorbed almost quantitatively at high temperatures, and it does not undergo decomposition inside the carbon matrix upon MW irradiation. It appears to be desorbed in a molecular state and then partially decomposed in the gas phase. This fact reduces the formation of deposits inside the porous network of the carbon, resulting in a better performance towards adsorption in successive cycles and favoring better regeneration efficiencies over the cycles.

The obtained results are also extremely interesting from an economic point of view; microwave technology opens the door to explore the possibility of recovery of the pollutant and sub-products, both intermediate compounds of pharmaceutical industry.

\section{Acknowledgment}

The authors thank Química Farmacéutica Bayer for kindly providing the adsorbent and for financial support. COA thanks the CSIC I3P Program, co-financed by the European Social Fund, for a postdoctoral contract.

\section{REFERENCES}

Ania, C.O. Parra, J.B. Menendez, J.A. Pis, J.J. 2004a. Microwave-induced regeneration of activated carbons polluted with phenol. A comparison with conventional thermal regeneration, Carbon, 42, 1377-1381. 
Ania, C.O. Parra, J.B. Pis, J.J. 2004b. Oxygen-induced Decrease in the Equilibrium Adsorptive Capacities of Activated Carbons. Ads. Sci. Technol. 22, 337-351.

Ania, C.O. Parra, J.B. Menendez, J.A. Pis, J.J. 2005a. Effect of microwave and conventional regeneration on the microporous and mesoporous network and on the adsorptive capacity of activated carbons, Micro. Meso. Mater. 85, 7-15.

Ania, C.O. Parra, J.B. Pevida, C. Arenillas, A. Rubiera, F. Pis, J.J. 2005b. Pyrolysis of activated carbons exhausted with organic compounds, J. Anal. Appl. Pyrol., 74, 518524.

Ayscough, N.J. Fawell, J. Franklin, G. Young, W. 2000. Review of Human Pharmaceuticals in the Environment, R\&D Technical Report P390, UK Environment Agency, Rio House, Bristol, ISBN 185705411 3, pp. 110

Bandosz, T.J. (Ed.) 2006. Activated carbon surfaces in environmental remediation. In the series Interface science and technology, Ed. Elsevier, New York.

Bathen, D. 2003. Physical waves in adsorption technology-an overview, Sep. Purif. Technol. 33, 163-177.

Bradshaw, S.M. Van Wyk, E.J. De Swardt, J.B. 1997. Preliminary economic assessment of microwave regeneration of activated carbon for the carbon in pulp process. International Microwave Power Institute. J. Microw. Power Electromagn. Energy, $32,131-144$.

Brunauer, B. Deming, L.S. Deming, W.E. Teller, E. 1940. On a theory of the van der Waals adsorption of gases, J. Am. Chem. Soc., 62, 1723-1732.

Cha, C.Y. Carlisle, C.T. 2001. Microwave process for volatile organic compound abatement. J. Air Waste Manage. Assoc. 51, 1628-1641.

Coss, M. Cha, C.Y. 2000. Microwave Regeneration of Activated Carbon Used for Removal of Solvents from Vented Air, J. Air Waste Manage. Assoc. 50, 529-535. 
Daughton, C.G. Ternes, T.A. 1999. Pharmaceuticals and personal care products in the environment: agents of subtle change?. Environ. Health Perspect, 107, 907-938.

Ernst, M. Jekel, M. 1999. Advanced treatment combination for groundwater recharge of municipal wastewater by nanofiltration and ozonation, Water Sci. Technol. 40, 277284.

Ferrari, B. Mons, R. Villat, B. Fraysse, B. Paxéus, N. Lo Guidice, R. Pollio, A. Garric, J. 2004. Environmental risk assessment of six human pharmaceuticals: Are the current environmental risk assessment procedures sufficient for the protection of the aquatic environment?. Environ. Toxicol. Chem. 23, 1344-1354.

Halling-Sorensen, B. Nielson, S.N. Lanzky, P.F. Ingerslev, F. Holten-Lutzhoft, J. Jorgensen, S.E. 1998. Ocurrence fate and effects of pharmaceutical substances in the environment- A review. Chemosphere, 35, 357-393.

Hensley, S. Murray, S. 2000. Use of samples in drug industry raises concern, Wall St. J. B1, B4.

Hernando, M.D. Mezcua, M. Fernandez-Alba, A.R. Barcelo, D. 2006. Environmental risk assessment of pharmaceutical residues in wastewater effluents, surface waters and sediments, Talanta 69, 334-342

Jones O.A. Lester, J.N. Voulvoulis, N. 2005. Pharmaceuticals: a threat to drinking water?. Trends Biotechnol., 23, 163-167.

Jones, D.A. Lelyveld, T.P. Mavrofidis, S.D. Kingman, S.W. Miles, N.J. 2002. Microwave heating applications in environmental engineering — a review. Resources Conserv. Recycl. 34, 75-90.

Kolpin, D.W. Furlong, G.E. Meyer, M. Thurman, E.M. Zaugg, S.D. Barber, LB. Buxton, H.T. 2002. Pharmaceuticals, Hormones, and Other Organic Wastewater 
Contaminants in U.S. Streams, 1999-2000: A National Reconnaissance, Environ. Sci. Technol. 36, 1202-1211.

Ku, H.S. Siores, E. Taube, A. Ball, J.A.R. 2002. Productivity improvement through the use of industrial microwave technologies. Computing Ind. Eng. 42, 281-290.

Liu, X. Quan, X. Bo, L. Chen, S. Zhao, Y. 2004. Simultaneous pentachlorophenol decomposition and granular activated carbon regeneration assisted by microwave irradiation, Carbon, 42, 415-422.

Liu, X. Yu, G. 2006. Combined effect of microwave and activated carbon on the remediation of polychlorinated biphenyl-contaminated soil, Chemosphere, 63, 228235.

Noh, J.S. Schwarz, J.A. Estimation of the point of zero charge of simple oxides by mass titration, J. Colloid Interf. Sci., 1989, 130 (1), 157-164.

Price, D.W. Schmidt, P.S. 1997. Microwave regeneration of adsorbents at low pressure: Experimental kinetics studies. J. Microw. Power Electromagn. Energy 32, 145-154.

O’Neil, M.J. Smith, A. Heckelman, P.E. Budavari, S. 2001. Merck Index: An Encyclopedia of Chemicals, Drugs, and Biologicals, John Wiley \& Sons; $13^{\text {th }}$ ed.

Quan, X. Liu, X. Bo, L. Chen, S. Zhao, Y. Cui, X. 2004. Regeneration of acid orange 7exhausted granular activated carbons with microwave irradiation, Wat. Res. 38, 4484-4490.

Radovic, L.R. Moreno-Castilla, C. Rivera-Utrilla, J. 2000. Carbon materials as adsorbents in aqueous solutions. (Radovic, L.R., Editor. Ed. Marcel Dekker) Chem. Phys. Carbon, 27, Ch. 4, 227-4.

Rao, A.M. Fung, A.W.P. Dresselhaus, M.S. Endo, M. 1996. Structural characterization of heat-treated activated carbon fibers. J. Mater. Res. 7, 1788-1794. 
Ruiz-Martínez, J. Ramos-Fernandez, E.V. Sepúlveda-Escribano A. Rodríguez-Reinoso F. 2006. Effect of thermal treatments on the surface chemistry of oxidized activated carbons, Stud. Surf. Sci. Catal., 160, 129-136.

Vogna D. Marotta, R. Andreozzi, R. Napoletano, A. d'Ischia, M. 2004. Kinetic and chemical assessment of the $\mathrm{UV} / \mathrm{H}_{2} \mathrm{O}_{2}$ treatment of antiepileptic drug carbamazepine, Chemosphere 54, 497-505.

Zhang-Steenwinkela, Y. van der Zandea, L.M. Castricuma, L. Blieka, A. van den Brinkb, R.W. Elzinga, G.D. 2005. Microwave-assisted in-situ regeneration of a perovskite coated diesel soot filter, Chem. Eng. Sci. 60, 797-804. 
Table 1. Adsorption capacities for salicylic acid on the as-received -CV-and heat treated carbon with microwave irradiation before -CV MW- and after successive cycles of regeneration. Salicylic acid initial concentration $2 \mathrm{~g} \mathrm{~L}^{-1}$. Columns packed with ca. $5 \mathrm{~g}$ of activated carbon

\begin{tabular}{|c|c|c|c|}
\hline & \multicolumn{3}{|c|}{ Adsorptive capacity [ $\mathrm{mg} \mathrm{g}^{-1}$ ] } \\
\hline \multirow[t]{3}{*}{$\mathrm{CV}$} & 372 & & \\
\hline & N4 & N8 & $C 8$ \\
\hline & $\left(450^{\circ} \mathrm{C}, \mathrm{N}_{2}\right)$ & $\left(850^{\circ} \mathrm{C}, \mathrm{N}_{2}\right)$ & $\left(850^{\circ} \mathrm{C}, \mathrm{CO}_{2}\right)$ \\
\hline CV MW & 388 & 409 & 428 \\
\hline Cycle 1 & 353 & 375 & 392 \\
\hline Cycle 2 & 329 & 371 & 390 \\
\hline Cycle 3 & 304 & 361 & 385 \\
\hline Cycle 4 & 281 & 351 & 379 \\
\hline Cycle 5 & 256 & 339 & 374 \\
\hline Cycle 6 & 233 & 326 & 366 \\
\hline
\end{tabular}


Table 2. Chemical composition, $\mathrm{pH}_{\mathrm{PZC}}$ and textural characteristics of the series of activated carbons obtained from the physical adsorption of nitrogen at $-196{ }^{\circ} \mathrm{C}$

\begin{tabular}{lcccccc}
\hline & $\begin{array}{c}S_{B E T} \\
\left(\mathrm{~m}^{2} \mathrm{~g}^{-1}\right)\end{array}$ & $\begin{array}{c}\boldsymbol{V}_{\text {micro }} \\
\left(\mathrm{cm}^{3} \mathrm{~g}^{-1}\right)\end{array}$ & $\begin{array}{c}\boldsymbol{V}_{\text {meso }} \\
\left(\mathrm{cm}^{3} \mathrm{~g}^{-1}\right)\end{array}$ & $\begin{array}{c}\boldsymbol{V}_{\mathrm{CO} 2}{ }^{\circ} \\
\left(\mathrm{cm}^{3} \mathrm{~g}^{-1}\right)\end{array}$ & $\begin{array}{c}\text { Oxygen } \\
{[\text { wt. \%] }}\end{array}$ & pH PZC \\
\hline CV & 1424 & 0.355 & 0.530 & 0.169 & 9.7 & 2.2 \\
\hline CVM W4 & 1365 & 0.346 & 0.468 & 0.163 & 6.5 & 3.3 \\
N4 cycle 3 & 769 & 0.199 & 0.314 & 0.168 & 6.0 & 3.8 \\
N4 cycle 6 & 428 & 0.080 & 0.289 & 0.139 & 5.8 & 3.9 \\
\hline CVM W N8 & 1065 & 0.306 & 0.417 & 0.173 & 4.1 & 4.2 \\
N8 cycle 1 & 1324 & 0.286 & 0.485 & 0.173 & 3.5 & 5.3 \\
N8 cycle 3 & 1042 & 0.263 & 0.390 & 0.154 & 3.2 & 8.1 \\
N8 cycle 6 & 813 & 0.202 & 0.337 & 0.142 & 2.1 & 9.1 \\
\hline CVM W C8 & 1346 & 0.345 & 0.430 & 0.236 & 3.7 & 7.3 \\
C8 cycle 1 & 1162 & 0.306 & 0.381 & 0.173 & 3.3 & 5.5 \\
C8 cycle 3 & 1124 & 0.304 & 0.370 & 0.169 & 2.9 & 8.3 \\
C8 cycle 6 & 915 & 0.241 & 0.233 & 0.153 & 2.3 & 9.2 \\
valuated from DFT applied to $\mathrm{N}_{2}$ adsorption at -196 ${ }^{\circ} \mathrm{C}$ & & \\
evaluated from DR method applied to $\mathrm{CO}_{2}$ adsorption at 0 ${ }^{\circ} \mathrm{C}$. & &
\end{tabular}


Figure 1. Regeneration efficiency of the series of activated carbons under different operating conditions.

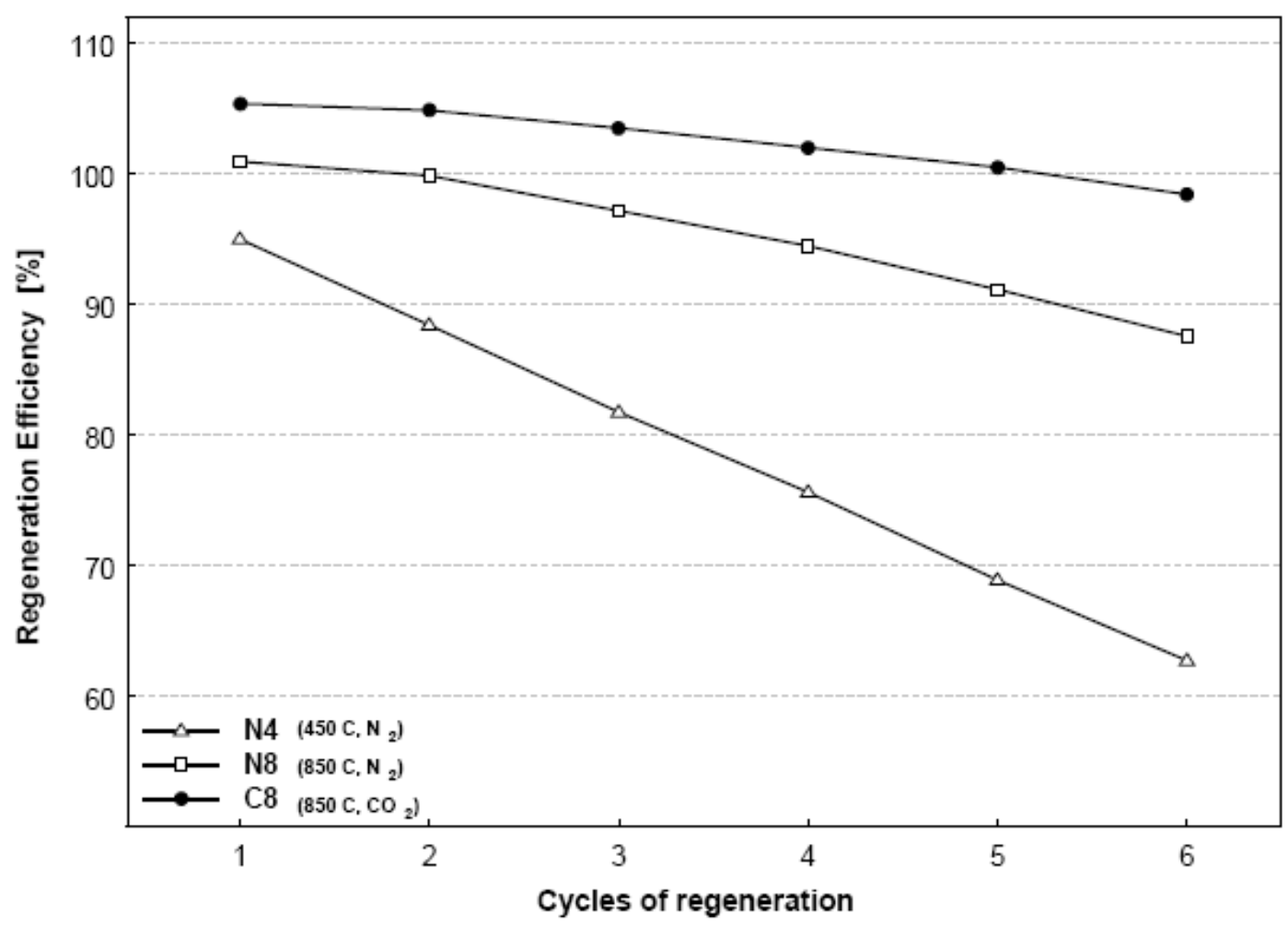


Figure 2. Stripping efficiency of the regenerated activated carbon under different operating conditions.

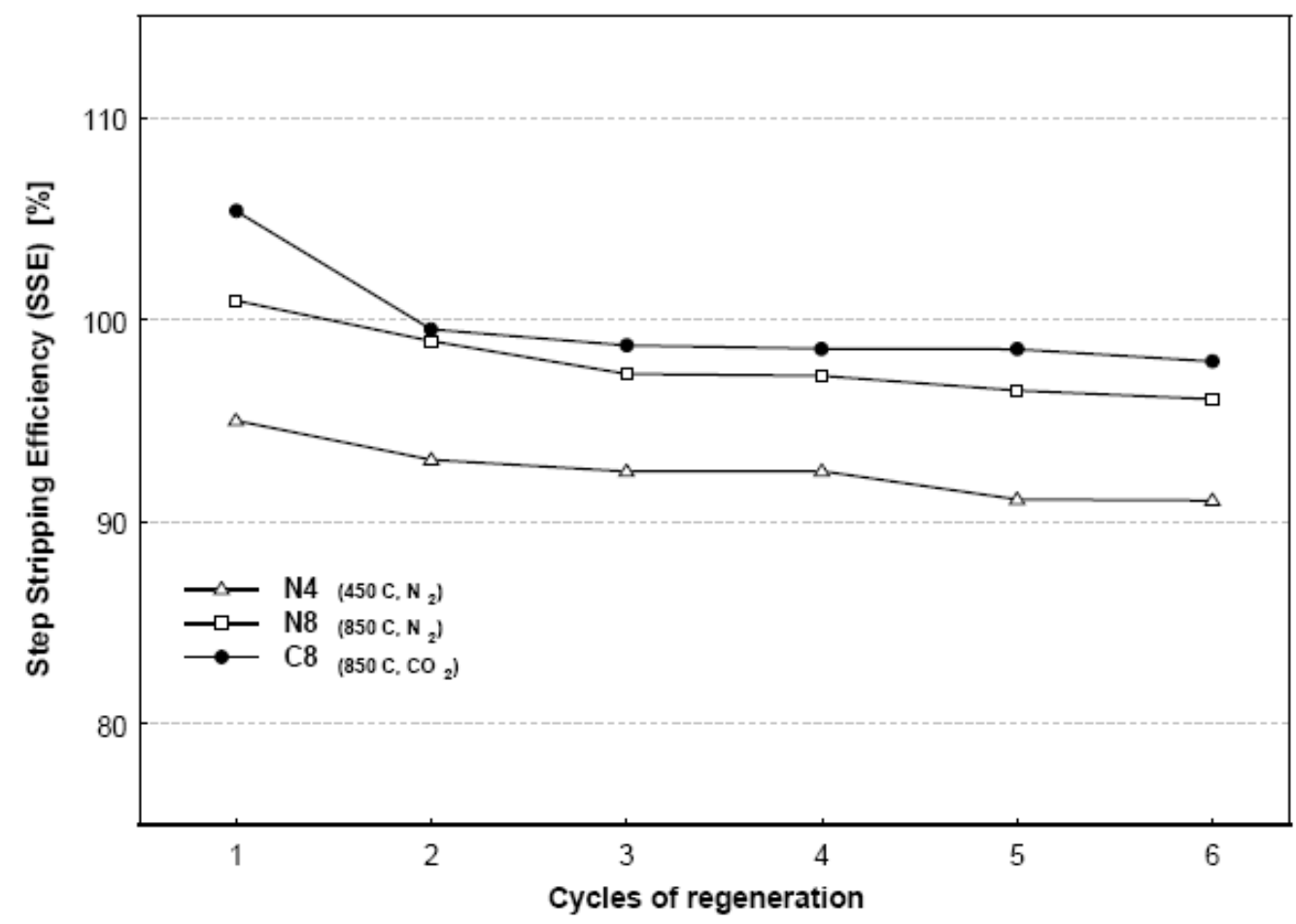


Figure 3. DTG profiles in nitrogen of the as-received carbon after microwave irradiation before (A) and after (B) salicylic acid adsorption (sat series).
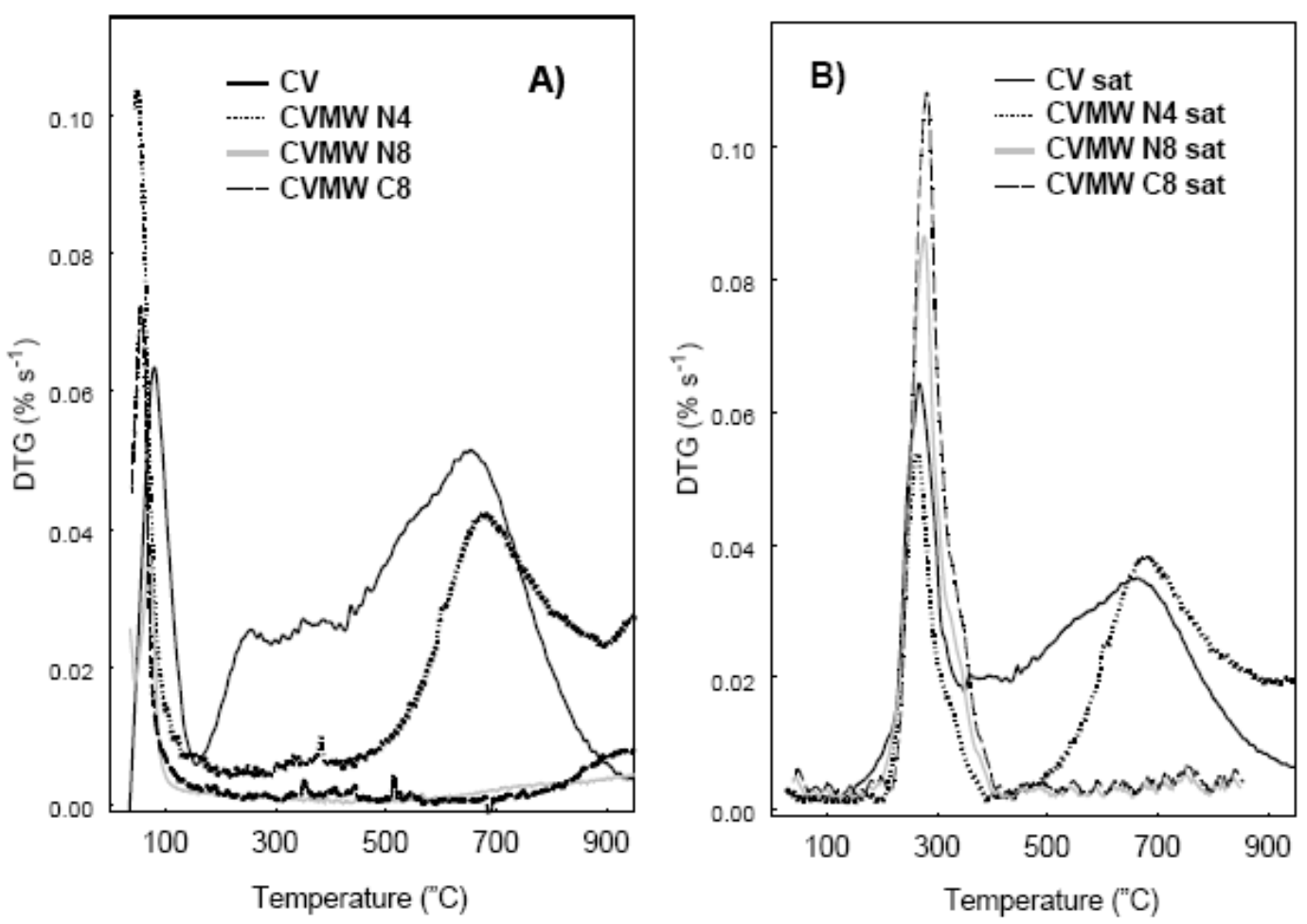
Figure 4. $\mathrm{CO}_{2}$ adsorption isotherms at $0{ }^{\circ} \mathrm{C}$ of the as-received carbon after microwave irradiation in absence of adsorbate (A) and after several cycles of regeneration (B-D).
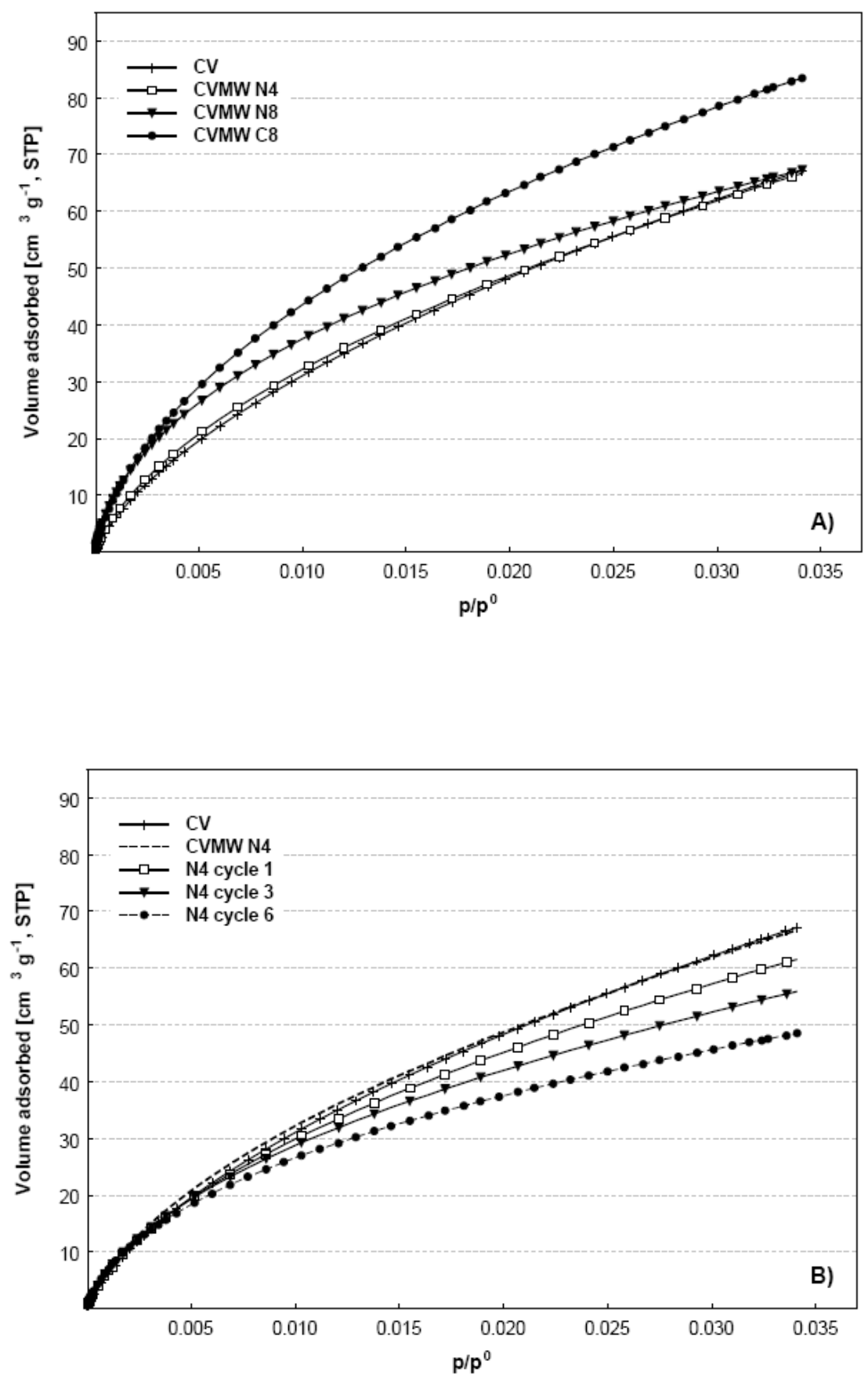

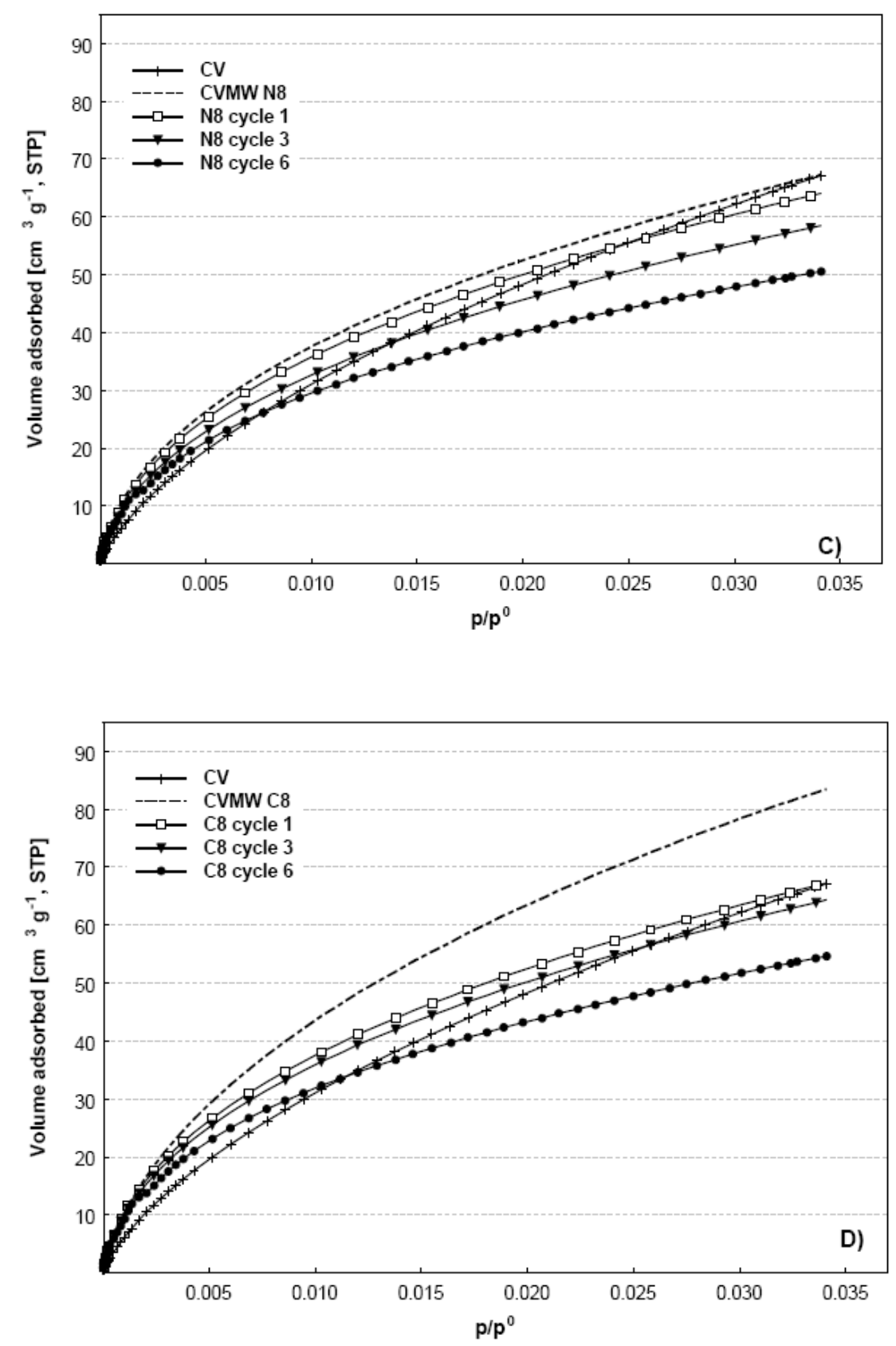